\title{
Social Vulnerability Indices as a Risk Factor for Heart Failure Readmissions
}

\author{
Manjari Rani Regmi, MD; Nitin Tandan, MD; Priyanka Parajuli, MD; Mukul Bhattarai, MD, MPH, FACP; \\ Ruby Maini, MD; Abhishek Kulkarni, MD; and Robert Robinson MD, MS, FACP
}

Objectives: The objective of our study was to use the parameters of social vulnerability index (SVI) to observe their association with the 30-day hospital readmissions in the heart failure population.

Methods: Data required for analysis were extracted from the electronic medical record. The geographic SVI data was then merged with the clinical data. Qualitative variables and reported as frequency and quantitative variables and reported as the mean \pm standard deviation. Variables from univariate analysis with a $P$ value of $\leq 0.10$ were evaluated using multivariate logistic regression with stepwise backward variable selection and receiver operating curve (ROC) analysis.

Results: The odds ratio of readmission predicted by HOSPITAL score was I.I37 ( $P$ value $=0.004$, $95 \% \mathrm{Cl}=1.04 \mathrm{I}-\mathrm{I} .24 \mathrm{I})$. SVI parameter recording disability showed odds ratio of I.52I ( $P$ value $=0.006$, $95 \% \mathrm{Cl}=1.125-2.058)$ and SVI parameter tracking vehicle ownership showed odds ratio of $15.355(P$ value $=0.014,95 \% \mathrm{Cl}=1.755-134.383$ ). The ROCs were generated for three scenarios: (i) HOSPITAL score only which had area under the curve (AUC) of 0.702 ( $P$ value $=0.015)$, (ii) SVI indicators tracking vehicle ownership and disability resulted in the AUC of $0.589(P$ value $=0.016)$, and (iii) all of the above combined increased the AUC increased to 0.718 ( $P$ value $=0.015)$.

Conclusions: Two social parameters (limited vehicle access and prevalence of disability) from the SVI showed a strong association with 30-day hospital readmissions.

Keywords: 30 -day readmission; Heart Failure; SVI; HOSPITAL score; Disability; No vehicle

$\mathrm{H}$ ospital readmissions reflect the quality of care provided by the health institutions. Increased 30-day readmission rates are not only indicators of poor quality of care but are also associated with increased healthcare costs., ${ }^{1,2}$ Among many other comorbidities, congestive heart failure is one of the top causes of hospital readmissions. ${ }^{3}$ Healthcare costs due to heart failure readmissions continue to afflict many hospitals across the world. In the United States alone, approximately one million heart failure readmissions were noted in $2014 .{ }^{4}$
While guideline-directed medical therapy is the mainstay of treating heart failure, social determinants of health have slowly been recognized as important factors that contribute to hospital readmission rates, morbidity, and 90-day mortality. ${ }^{5}$ More recently, the REGARDS study evaluated the influence of social determinants of health (including age, gender, education, health literacy, and social support) on 90-day mortality outcomes after hospitalization for heart failure. Active efforts have been concentrated to develop effective strategies and
Corresponding Author: Manjari Rani Regmi, MD, 70 I North First Street, Springfield, IL 6278I, Phone: 470-265- I046, Fax: 279-20I-6023,

Email:mregmi8I@siumed.edu; manjariregmi08.mr@gmail.com
Received: July 16, 2020

Revised: January 5, 2021

Accepted: April 16, 202।

doi: $10.3121 / \mathrm{cmr} .2021 .1603$ 
update existing ones to develop effective 30-day hospital readmissions predictive tools. Examples of some of these tools used to predict 30-day readmissions include HOSPITAL score, ${ }^{6}$ LACE index, ${ }^{7}$ and RAHF score. ${ }^{8}$ HOSPITAL Score was validated by Donzé et $\mathrm{al}^{6}$ and consists of seven components from which the acronym is formed: Hemoglobin at discharge, discharge from Oncology service, Sodium level at discharge, Procedure performed, Index admission Type (elective vs. emergent), number of previous hospital Admissions, and Length of stay $\geq 5$ days. Although these scores consider patients' characteristics and co-morbidities, most of them do not include social determinants of health like poverty, disability, access to health care, and so forth. In an attempt to gaze into social determinants affecting 30-day readmissions, we used the Social Vulnerability Index (SVI) and its parameters to predict readmissions and compare the results with those from the existing tools.

SVI is a tool to measure the resilience of the communities when confronted by external stresses on human health, natural or human-caused disasters, or disease outbreaks. The index includes different parameters under four themes: (i) socioeconomic status, (ii) household composition and disability, (iii) minority status and language, and (iv) housing and transportation. A total of 15 variables are used under these 4 themes. ${ }^{9}$ The SVI index has been seen to be not only beneficial in planning for the preparedness of natural disasters, but it can also provide useful information regarding health outcomes at a community level. ${ }^{10}$

This study primarily aims to identify the association of SVI parameters with the 30-day readmitted subgroup of patients

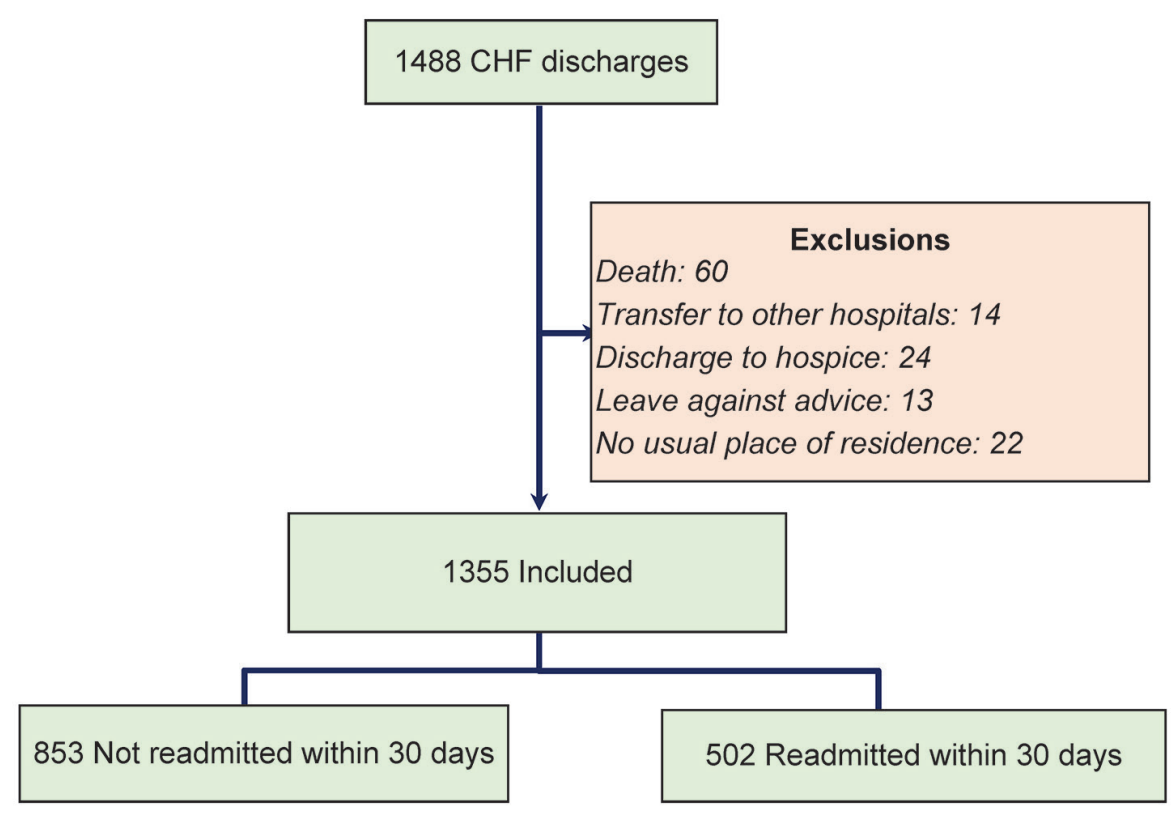

Figure 1. Study flow diagram for 30-day readmission. discharged from congestive heart failure. A secondary goal is to compare the predictive power of these parameters with those of the HOSPITAL score.

\section{Materials and Methods}

The study design was a retrospective cohort study. The study population included all adult medical patients discharged from the Southern Illinois University School of Medicine (SIU-SOM) hospitalist service at Memorial Medical Center from June 2017 to June 2019. We performed the study on this population to determine if components of the geographic SVI were useful predictors of any cause (avoidable and unavoidable) hospital readmission within 30 days. Exclusion criteria were not having a usual place of residence, being transferred to another acute care hospital, leaving the hospital against medical advice, or in-hospital death (Figure 1). The study endpoint was all-cause readmission to the same hospital within 30 days. All-cause readmission was selected to be in sync with the measure used by the Medicare Hospital Readmission Reduction Program.

Memorial Medical Center is a 507-bed not-for-profit universityaffiliated tertiary care center located in Springfield, Illinois, USA. The SIU-SOM hospitalist service is the teaching service for the internal medicine residency program. Faculty who staff the hospitalist service are board certified or board eligible internal medicine physicians with a hospital-based practice. Patients are generally admitted from the hospital emergency department or transferred from other regional hospitals with acute medical illnesses. Elective hospital admissions are extremely rare for this hospitalist service.

De-identified data for age, gender, vital signs, length of hospital stay, International Classification of Disease (ICD) codes, number of emergency department (ED) visits in the last 6 months, readmission status at 30 days after discharge, and other information needed to calculate the HOSPITAL score, LACE index, and RAHF score were extracted. Missing laboratory data required for the HOSPITAL score were coded in the normal range. Also, the ZIP codes of where the patients lived were obtained from the electronic health record to gather the geographic SVI data for the corresponding community. The extracted outcomes for the 15 social parameters were binary ( 0 or 1$)$, where 1 indicates the community is below the $90^{\text {th }}$ percentile in the nation in that respective category. ${ }^{9}$ The geographic SVI data were then merged with the clinical data. The ZIP code fields were then deleted to create a completely de-identified data 
Table 1. Univariate Analysis

\begin{tabular}{|c|c|c|c|}
\hline Characteristic & $\begin{array}{l}\text { Not Readmitted } \\
\qquad(\mathrm{N}=853)\end{array}$ & $\begin{array}{l}\text { Readmitted } \\
\text { (N = 502) }\end{array}$ & $P$ Value \\
\hline Mean age (SD) & $71(14)$ & $67(13)$ & $<0.001$ \\
\hline Female & 389 (46\%) & $300(60 \%)$ & $<0.001$ \\
\hline African American race & 85 (10\%) & $88(18 \%)$ & $<0.001$ \\
\hline \multicolumn{4}{|l|}{ SVI parameters } \\
\hline Per capita income & $100(12 \%)$ & 95 (19\%) & $<0.001$ \\
\hline Disability & $249(29 \%)$ & $233(46 \%)$ & $<0.001$ \\
\hline No vehicle & $1(<1 \%)$ & $10(2 \%)$ & $<0.001$ \\
\hline Multiunit housing & $2(<1 \%)$ & $10(2 \%)$ & 0.001 \\
\hline Group quarters & $192(23 \%)$ & $151(30 \%)$ & 0.002 \\
\hline Age $>65$ & $158(19 \%)$ & $125(25 \%)$ & 0.006 \\
\hline Age $<17$ & $19(2 \%)$ & $5(1 \%)$ & 0.134 \\
\hline Single parent household & $1(<1 \%)$ & 0 & 1.00 \\
\hline Minority & 0 & 0 & \\
\hline Limited English & $18(2 \%)$ & $5(1 \%)$ & 0.190 \\
\hline Mobile home & $155(18 \%)$ & $77(15 \%)$ & 0.204 \\
\hline Crowded housing & $9(1 \%)$ & 0 & 0.031 \\
\hline No high school diploma & $1(<1 \%)$ & 0 & 1.00 \\
\hline \multicolumn{4}{|l|}{ Readmission Scores } \\
\hline HOSPITAL score & $3.55(1.7)$ & $5.09(2.1)$ & $<0.001$ \\
\hline LACE index & $10.54(2.3)$ & $11.71(2.4)$ & $<0.001$ \\
\hline RAHF score & $24.40(9.1)$ & $28.04(9.5)$ & $<0.001$ \\
\hline Charlson Comorbidity Index & $3.08(1.2)$ & $3.40(1.1)$ & $<0.001$ \\
\hline \multicolumn{4}{|l|}{ Medical Comorbidities } \\
\hline Diabetes without complications & $255(30 \%)$ & 200 (40\%) & $<0.001$ \\
\hline Diabetes with complications & $244(29 \%)$ & $232(46 \%)$ & $<0.001$ \\
\hline Renal disease & $314(37 \%)$ & $269(54 \%)$ & $<0.001$ \\
\hline Mild liver disease & $54(6 \%)$ & $56(11 \%)$ & 0.002 \\
\hline Moderate to severe liver disease & $17(2 \%)$ & $26(5 \%)$ & 0.002 \\
\hline Myocardial infarction & $191(22 \%)$ & $87(17 \%)$ & 0.026 \\
\hline Electrolyte abnormalities & $361(42 \%)$ & $238(47 \%)$ & 0.070 \\
\hline Stroke & $59(7 \%)$ & $23(5 \%)$ & 0.098 \\
\hline Dementia & $70(8 \%)$ & $41(8 \%)$ & 1.00 \\
\hline Chronic lung disease & $429(50 \%)$ & $268(53 \%)$ & 0.285 \\
\hline Connective tissue disease & $33(4 \%)$ & $13(3 \%)$ & 0.277 \\
\hline Peptic ulcer disease & $14(2 \%)$ & $6(1 \%)$ & 0.643 \\
\hline Peripheral artery disease & $97(11 \%)$ & $55(11 \%)$ & 0.859 \\
\hline Paralysis & $25(3 \%)$ & $11(1 \%)$ & 0.468 \\
\hline Cancer & $61(7 \%)$ & $36(7 \%)$ & 1.00 \\
\hline Metastatic cancer & $30(2 \%)$ & $16(1 \%)$ & 0.877 \\
\hline Substance abuse & $23(3 \%)$ & $9(2 \%)$ & 0.356 \\
\hline
\end{tabular}


file that was provided to the researchers for analysis. The de-identified nature of the data makes accurate linkage to other data sources impossible. One of the predictors in the HOSPITAL score is admission to an oncology service. However, the study hospital does not have oncology admitting service. The local practice pattern is to have hospitalists admit oncology patients for inpatient care. Therefore, patients with oncology-related ICD codes were considered to have been discharged from an oncology service. As this is a single-center study, readmissions to other hospitals could not be detected. From the compiled dataset, the HOSPITAL score, Charlson score, RAHF score, and LACE index were calculated for each admission. Medical comorbidities were determined based on documented ICD-10 codes in the manner specified for the Charlson score and the RAHF score.

Institutional review board review for this study was obtained from the Springfield Committee for Research Involving Human Subjects. This study was determined not to meet the criteria for research involving human subjects according to 45 CFR 46.101 and 45 CFR 46.102.

\section{Statistical Analysis}

SVI components at the $90^{\text {th }}$ percentile of national prevalence for the ZIP code of patient residence were investigated as potential predictors of all-cause hospital readmission (Table 1). This measure was selected because it is characterized as an indicator of very high SVI. The Pearson chi $^{2}$ or Fisher's exact test were used when evaluating qualitative variables and reported as frequency (\%). The Mann-Whitney U or KruskalWallis tests were used to evaluate quantitative variables and reported as the mean \pm standard deviation. Variables from univariate analysis with a $P$ value of $\leq 0.10$ were evaluated using multivariate logistic regression with stepwise backward variable selection and receiver operating curve (ROC) analysis. Exploratory analysis using SVI components shown to be significant predictors of hospital readmission in multivariate analysis was then conducted. For each significant SVI predictor, an additional point was added to the HOSPITAL score to determine if this resulted in improved performance. For calculating the ROCs, HOSPITAL scores of 0-4 points were classified as low risk for readmission (5\%), 5-6 points intermediate risk $(10 \%)$, and 7 or more points as high risk (20\%) based on the initial validation study of the HOSPITAL score. ${ }^{6}$ SPSS version 25 (SPSS Inc., Chicago, IL, USA) was used for statistical analysis. Two-sided $P$ values $<0.05$ were considered significant.

\section{Results}

Out of 1488 discharges, 133 were excluded based on the exclusion criteria. The eligible sample size was 1355 (Figure 1). The sample was almost evenly divided between males $(\sim 49 \%)$ and females $(\sim 51 \%)$. The sample was separated into two groups - the first group consisted of patients who were not readmitted within 30 days, and the second group consisted of patients who were readmitted within 30 days. Table I summarizes the statistics of various qualitative and quantitative variables of 1355 discharged patients. The 30 -day readmission rate was $37 \%$. The male to female ratio in the readmitted group was 40/60, whereas it was $46 / 54$ in the non-readmitted group $(P$ value $=0.001)$. When segmented based on race, the study population comprised $12 \%$ African Americans. The percentage of African Americans in the readmitted and non-readmitted group was $18 \%$ and $10 \%$, respectively $(P$ value $=0.001)$.

In addition to demographics, Table 1 also shows the parameters of the SVI index. Few important observations are presented here. The readmitted group consisted of $46 \%$ of the population belonging to the ZIP codes with greater than $90^{\text {th }}$ percentile disability compared to $29 \%$ in the non-readmitted group $(P$ value $<0.001)$. For reference, $35 \%$ of the overall study population belonged to such ZIP codes. Another parameter is belonging to the ZIP codes with significantly low vehicle ownership ( $>90^{\text {th }}$ percentile); $0.8 \%$ (11) of the study population belonged to such ZIP codes. It was observed that the percentage of this population in the non-readmitted and readmitted group was $0.12 \%$ and $2 \%$, respectively ( $P$ value $<0.001)$. Table 1 also shows the readmission scores of various scoring tools. All tools (HOSPITAL, LACE, RAHF, and Charlson) demonstrated good predictive power with $P$ values $<0.001$.

Table 1 lists multiple comorbidities associated with increased readmission. Diabetes, both with and without complication, was statistically more prevalent in the readmitted group compared to the non-readmitted group. Diabetes with complications was present in $46 \%$ of the readmitted patients, and diabetes without complications was present in $40 \%$ of the readmitted patients $(P$ value $=0.01)$. Renal disease was found in $54 \%$ of patients in the readmitted group compared to $37 \%$ of patients in the non-readmitted group ( $P$ value $=0.001)$. Further, $47 \%$ of patients in the readmitted group had some electrolyte abnormalities, whereas $42 \%$ had these abnormalities in the non-readmitted group $P$ value $=0.070$ ). Underlying comorbidities of mild and moderate to severe liver disease were present in relatively higher percentages in the readmitted group (11\% and 5\%) compared to the nonreadmitted group $(6 \%$ and $2 \%)$ with $P$ value $=0.002$. Interestingly, myocardial infarction was more common $(22 \%)$ in the non-readmitted group compared to $17 \%$ in the readmitted group $(P$ value $=0.026)$.

Odds ratios from multivariate analysis are shown in Table 2. The odds of readmission predicted by HOSPITAL score was $1.137(P$ value $=0.004,95 \% \mathrm{CI}=1.041-1.241) . \mathrm{SVI}$ parameter recording disability showed odds ratio of $1.521(P$ value $=0.006,95 \% \mathrm{CI}=1.125-2.058)$. Significantly high odds ratio was observed for the SVI parameter tracking vehicle ownership with odds ratio of $15.355(P$ value $=0.014$, $95 \%$ CI $=1.755-134.383)$. Renal disease and diabetes without complication had odds ratio of 2.080 ( $P$ value $=$ 
Table 2. Multivariate Analysis

\begin{tabular}{lccc}
\hline Characteristic & $\begin{array}{c}\text { Odds ratio for } \\
\text { Readmission }\end{array}$ & $95 \%$ Cl & $\boldsymbol{P}$ Value \\
\hline Age, mean (SD) & 0.986 & $0.976-0.997$ & 0.11 \\
Female & 0.664 & $0.502-0.879$ & 0.004 \\
African American race & 1.490 & $0.968-2.295$ & 0.070 \\
Hospital admissions (last year) & 2.354 & $2.031-2.728$ & $<0.001$ \\
ED visits (last 6 months) & 1.098 & $1.014-1.188$ & 0.021 \\
HOSPITAL score & 1.137 & $1.041-1.241$ & 0.004 \\
Stroke & 0.521 & $0.273-0.995$ & 0.048 \\
Diabetes (without complications) & 1.528 & $1.137-2.053$ & 0.005 \\
Renal disease & 2.080 & $1.562-2.768$ & $<0.001$ \\
Disability & 1.521 & $1.125-2.058$ & 0.006 \\
Crowded housing & 0.00 & & 0.998 \\
No vehicle & 15.355 & $1.755-134.383$ & 0.014 \\
\hline
\end{tabular}

above the $90^{\text {th }}$ percentile in the nation to which it belongs. This parameter has a strong correlation to 30-day readmission. Approximately $1 \%$ of the patient population in this study belonged to such communities, and more than $90 \%$ of this group were readmitted within the next 30 days. Although the size of this population segment in the sample was very low, ${ }^{11}$ it is certainly intriguing to observe the statistical significance due to an egregious odds ratio. Living in a community that has a lower percentage of households owning vehicles does not imply absolutely that a patient has no access to a vehicle. However, it increases the likelihood of the patient not owning a vehicle, thereby limiting access to healthcare facilities such as hospitals and pharmacies in a timely fashion or not being able to purchase fresh food, thereby

$0.001,95 \% \mathrm{CI}=1.562-2.768)$ and $1.528(P$ value $=0.001$, $95 \% \mathrm{CI}=1.137-2.053$ ) respectively.

Figure 2 shows the ROC of three sources: (i) HOSPITAL score, (ii) SVI indicators tracking vehicle ownership and disability, and (iii) both combined. HOSPITAL score had area under the curve (AUC) of $0.702(P$ value $=0.015,95 \% \mathrm{CI}$ $0.673-0.730)$. The two parameters of the SVI index resulted in the AUC of 0.589 ( $P$ value $=0.016,95 \%$ CI $0.557-0.621)$. When combining the HOSPITAL score with the abovementioned SVI indices, the AUC increased to 0.718 ( $P$ value $=0.015,95 \%$ CI $0.690-0.747$ ).

\section{Discussion}

Most studies on hospital readmissions focus on analyzing large medical datasets, but hospital readmissions are affected by multifactorial parameters including patient factors (medical diagnosis, health status, and existing comorbidities) and social factors. In this study, we aimed to identify whether several social factors, in addition to other medical factors, contribute to hospital readmissions and if true, quantify their effects.

Using the results of the SVI from the Centers for Disease Control and Prevention provided a quantifiable measure of fifteen social parameters. As highlighted by Grabovsch et $\mathrm{al}^{11}$ and Bergstrand et al, ${ }^{12}$ SVI can also be useful to identify and analyze situations other than preparedness for natural disasters. Further, Yee et $\mathrm{al}^{10}$ applied SVI to identify the need for teen pregnancy intervention in the United States. Similarly, Khan et $\mathrm{al}^{13}$ used this index to assess the health outcomes in South Carolina, and Gay et al $^{14}$ examined youth physical fitness using SVI. To the best of the authors' knowledge, this is the first reported study where SVI is used to identify 30-day hospital readmissions.

The SVI parameter of not having a vehicle tracks whether a community's percentage of household not owning vehicles is limiting a patient's quality of life. In a study, Warchol et al ${ }^{15}$ highlighted increased access to transportation as one of the potential social strategies to reduce hospital readmissions. Further, it also implies that the community might have an

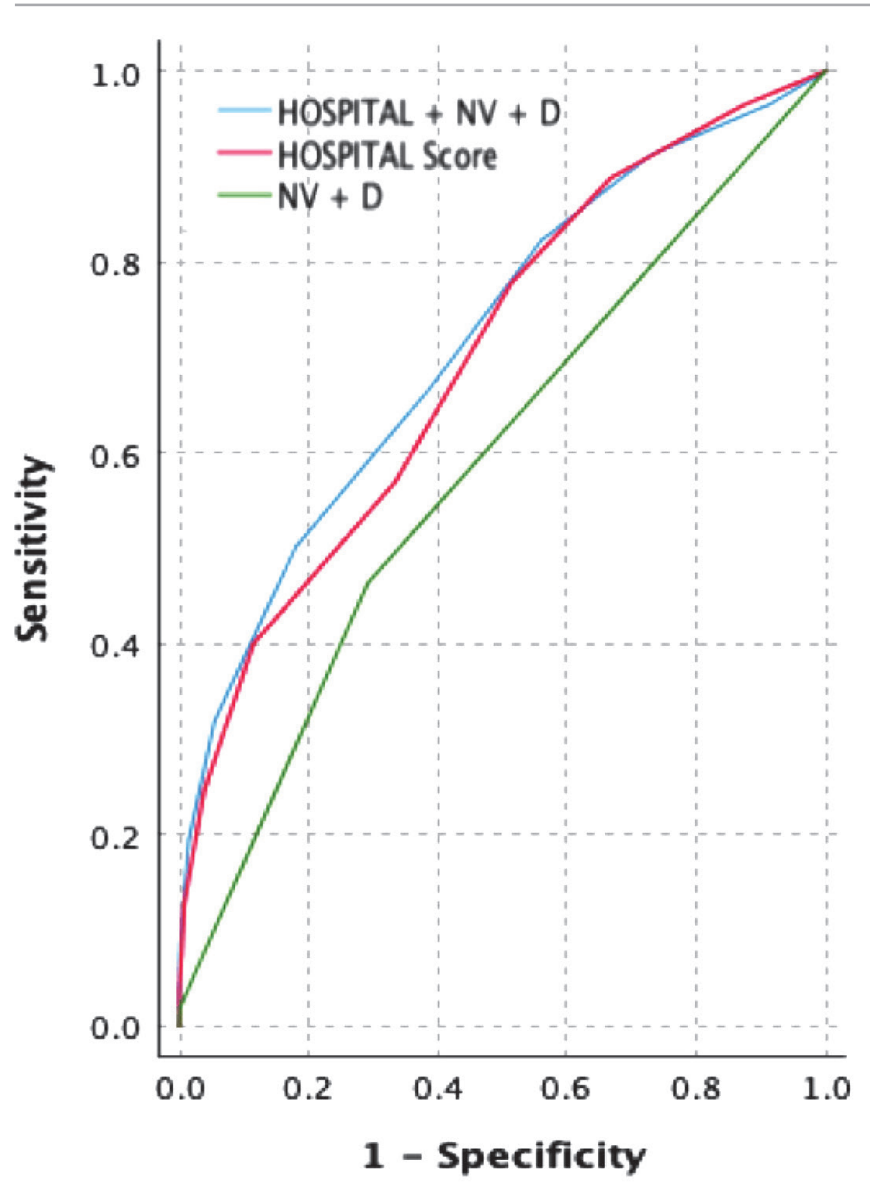

Figure 2. Receiver Operating Characteristic curve for three scenarios: Hospital score + No vehicle + Disability [blue], Hospital score [red], No vehicle + Disability [green]. 
overall lower economic standard. Studies by van Walraven et $\mathrm{al}^{16}$ and Silverstein et $\mathrm{al}{ }^{17}$ have shown that hospital readmissions are more common in patients belonging to low-income neighborhoods. Similarly, another SVI parameter tracking disability in the same way owning vehicles was tracked showed an increased likelihood for readmission. Although the correlation does not imply the patient is disabled, in the absence of other information, the likelihood remains. Studies by Ottenbacher et $\mathrm{al}^{18}$ and Meddings et $\mathrm{al}^{19}$ have indicated disability to be a risk factor for hospital readmissions.

Two social parameters (limited vehicle access and prevalence of disability) from the SVI offered strong indicators of hospital readmissions. We attempted to combine these parameters to an internationally validated HOSPITAL scoring tool to evaluate if a better prediction model could be achieved. From the ROC, we saw the incorporation of these two SVI indices led to an increase in the AUC and suggested an improvement in the strength of the readmission risk prediction. HOSPITAL score currently includes patient's medical factors such as hemoglobin at discharge, discharge from an oncology service, sodium level at discharge, procedures performed during the hospital stay, index of admission (elective vs. emergent), number of hospital admissions during the previous year, and length of stay. ${ }^{6}$ Supplementation of additional parameters from the SVI, which incorporated variables of the community, helped strengthen its predictability. The findings from this study suggest that SVI may offer great insights and correlations to improve the validity and accuracy of the readmission risk tools like the HOSPITAL score.

Some other findings were also notable. From the univariate analysis, it was apparent the risk of the African American population being readmitted was twice as high compared to the white population. This finding is not unique to this study; many studies have shown an increase in readmissions in the black population compared to their counterparts. A study conducted by Philbin et $\mathrm{al}^{20}$ concluded that in a congestive heart failure population, after adjusting to patient characteristics, black patients had significantly higher odds of readmission compared to white patients. Similarly, Joynt et $\mathrm{al}^{21}$ looked into the effects of race in readmissions for myocardial infarction, pneumonia, and heart failure and concluded that a black patient has a $13 \%$ higher chance of being readmitted compared to a white patient. The observed correlation in our study does not identify causality. However, it is consistent with the other aforementioned studies, and it merely identifies the differences that the African American community have experienced in the social sphere of US society.

Our analysis also identified certain medical comorbidities to be strong indicators for readmissions. Both diabetes and renal disease correlated with increased readmissions. These findings have also been reported in previous studies. In a study by Allaudeen et $\mathrm{al}^{22}$ patients with underlying renal disease showed an increased risk of 30-day readmissions compared to patients without renal disease. Similarly, from a multicenter study conducted by Donze et al, ${ }^{23}$ chronic kidney disease had a significantly higher risk of avoidable readmissions within 30 days. The same study, however, did not find any correlation regarding diabetes, in contrast to the observation from our study population.

\section{Limitations}

One of the primary limitations of our study is that we used relative parameters of SVI which relies on a comparative relationship with the national strength. Using this eliminates the absolute strength of the community. A second limitation of our study is the sample size, which was small. Larger sample sizes may be needed in the future to further validate this association. A less concerning limitation is that we could not include patients readmitted to other institutions. However, we believe those numbers would be a negligible fraction of the study population, since the hospital in the study is the only major hospital in the geographical area.

\section{Conclusion}

Several tools have emerged to accurately predict readmissions rates. While most of these tools utilize patients' clinical and demographic characteristics to compute readmission risk, this study analyzed the significance of incorporating social parameters as well. The social parameters in this study were obtained from data provided by the SVI. The primary finding of this study was that two parameters of the SVI-vehicle ownership, and prevalence of disability in the communityshowed a higher correlation to the 30 -day readmission rate. Higher predictive power for readmission rates was observed especially when combining these two indices with already validated HOSPITAL score. The results of this study shed light on the effect of resources as well as the community on the patient's overall improvement conditions, and it suggests incorporating social parameters when computing readmissions risk. Further research on a multi-center study is warranted to validate the evidence observed in this study.

\section{References}

1. Ross JS, Chen J, Lin Z, et al. Recent national trends in readmission rates after heart failure hospitalization. Circ Heart Fail. 2010;3(1):97-103.

2. Bernheim SM, Grady JN, Lin Z, et al. National patterns of risk-standardized mortality and readmission for acute myocardial infarction and heart failure. Update on publicly reported outcomes measures based on the 2010 release. Circ Cardiovasc Qual Outcomes. 2010;3(5):459467.

3. Iyngkaran P, Liew D, Neil C, Driscoll A, Marwick TH, Hare DL. Moving From Heart Failure Guidelines to Clinical Practice: Gaps Contributing to Readmissions in Patients With Multiple Comorbidities and Older Age. Clin Med Insights Cardiol. 2018;12. 
4. Centers for Disease Control and Prevention. Heart

Disease: Heart Failure. Available at: cdc.gov. https://www. cdc.gov/heartdisease/heart_failure.htm. Accessed June 8, 2020.

5. Sterling MR, Ringel JB, Pinheiro LC, et al. Social Determinants of Health and 90?Day Mortality After Hospitalization for Heart Failure in the REGARDS Study. J Am Heart Assoc. 2020;9(9):e014836.

6. Donzé JD, Williams MV, Robinson EJ, et al. International Validity of the HOSPITAL Score to Predict 30-Day Potentially Avoidable Hospital Readmissions. JAMA Intern Med. 2016;176(4):496-502.

7. van Walraven C, Dhalla IA, Bell C, et al. Derivation and validation of an index to predict early death or unplanned readmission after discharge from hospital to the community. CMAJ. 2010;182(6):551-557.

8. Chamberlain RS, Sond J, Mahendraraj K, Lau CSM, Siracuse BL. Determining 30-day readmission risk for heart failure patients: the Readmission After Heart Failure scale. Int J Gen Med. 2018;11:127-141.

9. Centers for Disease Control and Prevention. Agency for Toxic Substances and Disease Registry. CDC/ATSDR Social Vulnerability Index. Available at: https://www. atsdr.cdc.gov/placeandhealth/svi/index.html. Accessed June 8, 2020.

10. Yee CW, Cunningham SD, Ickovics JR. Application of the Social Vulnerability Index for Identifying Teen Pregnancy Intervention Need in the United States. Matern Child Health J. 2019;23(11):1516-1524.

11. Grabovschi C, Loignon C, Fortin M. Mapping the concept of vulnerability related to health care disparities: a scoping review. BMC Health Serv Res. 2013;13(1):94.

12. Bergstrand K, Mayer B, Brumback B, Zhang Y. Assessing the Relationship Between Social Vulnerability and Community Resilience to Hazards. Soc Indic Res. 2015;122(2):391-409.

13. Khan SS, Eberth JM, Emrich CT. Social vulnerability and its relationship with health outcomes in South Carolina. Ann Epidemiol. 2015;25(9):710.

14. Gay JL, Robb SW, Benson KM, White A. Can the Social Vulnerability Index Be Used for More Than Emergency Preparedness? An Examination Using Youth Physical Fitness Data. J Phys Act Health. 2016;13(2):121-130.

15. Warchol SJ, Monestime JP, Mayer RW, Chien WW. Strategies to Reduce Hospital Readmission Rates in a Non-Medicaid-Expansion State. Perspect Health Inf Manag. 2019;16(Summer):1a. eCollection Summer 2019.

16. van Walraven C, Wong J, Forster AJ. Influence of neighborhood household income on early death or urgent hospital readmission. J Hosp Med. 2013;8(5):261-266.

17. Silverstein MD, Qin H, Mercer SQ, Fong J, Haydar Z. Risk factors for 30-day hospital readmission in patients $\geq 65$ years of age. Proc (Bayl Univ Med Cent). 2008;21(4):363-372.
18. Ottenbacher KJ, Smith PM, Illig SB, Fiedler RC, Granger $\mathrm{CV}$. Length of stay and hospital readmission for persons with disabilities. Am J Public Health. 2000;90(12):19201923.

19. Meddings J, Reichert H, Smith SN, et al. The Impact of Disability and Social Determinants of Health on Condition-Specific Readmissions beyond Medicare Risk Adjustments: A Cohort Study. J Gen Intern Med. 2017;32(1):71-80.

20. Philbin EF, DiSalvo TG. Influence of race and gender on care process, resource use, and hospital-based outcomes in congestive heart failure. Am J Cardiol. 1998;82(1):7681.

21. Joynt KE, Orav EJ, Jha AK. Thirty-day readmission rates for Medicare beneficiaries by race and site of care. JAMA. 2011;305(7):675-681.

22. Allaudeen N, Vidyarthi A, Maselli J, Auerbach A. Redefining readmission risk factors for general medicine patients. J Hosp Med. 2011;6(2):54-60.

23. Donzé J, Lipsitz S, Bates DW, Schnipper JL. Causes and patterns of readmissions in patients with common comorbidities: retrospective cohort study. BMJ. 2013;347(dec16 4):f7171.

\section{Author Affiliations}

Manjari Rani Regmi, $M D^{*}$; Nitin Tandan, $M D^{*}$; Priyanka Parajuli, MD*; Mukul Bhattarai, MD, MPH, FACP*; Ruby Maini, MD*; Abhishek Kulkarni, MD*; and Robert Robinson, $M D, M S, F A C P^{*}$

*Southern Illinois University School of Medicine, Springfield, Illinois, USA 